\title{
UK Parkinson's Excellence Network: empowering service improvement across the UK
}

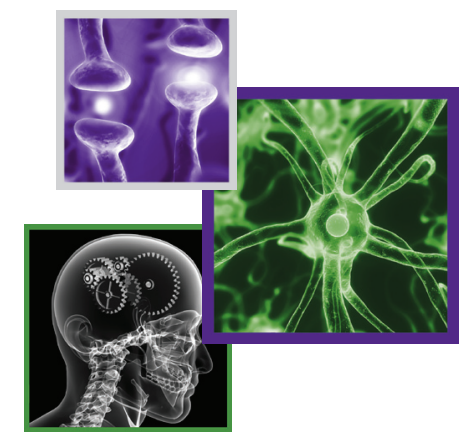

\author{
David Burn*
}

\section{Practice points}

- The Excellence Network is a one-stop-shop for Parkinson's collaboration, evidence, education and resources to drive service improvement.

- Key to the network is bringing together the resources and infrastructure of Parkinson's UK and the passion and commitment of professionals, underpinned by the voices of people with Parkinson's.

- The Excellence Network offers resources to support service improvement and engage people affected by Parkinson's, comprehensive information about education and training and collaboration opportunities. It also has the latest news, events and funding opportunities for professionals.

- It has six themed working groups, whose job is to focus on improvement in a particular subject area, such as education or service development.

- It also has 20 regional working groups, whose job is to empower and support people in their areas to drive change.

- There will be an oversight group to set the strategy and monitor the success of the network in driving positive change.

- The Network will also be able to draw on the valuable advice and experience of an external advisory group.

- The UK Parkinson's Excellence Network is an opportunity to take the best ideas from professionals all over the country to share and disseminate them more widely.

- The aim is to raise the bar so that every person with Parkinson's gets a high-quality standard of care, and as professionals, we can save time and effort by not 'reinventing the wheel'.

SUMMARY Parkinson's UK, together with leading Parkinson's professionals, has set up the UK Parkinson's Excellence Network to bring together the passion and expertise of leading clinicians with the strategic leadership and resources of Parkinson's UK underpinned by the voice of people affected by Parkinson's. Launched in London in February 2015, the Excellence Network aims to drive sustainable improvements in health and social care services. It will provide a more strategic approach to clinical development so that Parkinson's services across health and social care can be transformed to provide the best quality care across the UK.

\footnotetext{
*Institute of Neuroscience, Newcastle-upon-Tyne Hospitals NHS Foundation Trust, Newcastle-upon-Tyne, UK;

and

Parkinson's UK, 215 Vauxhall Bridge Rd, London SW1V 1EJ;

and

Biomedical Research Unit in Lewy Body Dementia \& Clinical Ageing Research Unit, Newcastle NIHR, Newcastle, UK;

david.burn@ncl.ac.uk
}

Future $\because$
Medicine $\%$ part of 


\section{KEYWORDS}

- collaboration

- interdisciplinary

- Parkinson's • service improvement $\bullet$ UK

Parkinson's audit • UK

Parkinson's Excellence Network

\section{Brief account of history/strategy}

In order to drive their new strategy, Parkinson's UK undertook a huge listening exercise to record the experiences of people affected by Parkinson's and the top three things that would change their lives with the condition. These findings shaped the main aims of the strategy, which were; to move as quickly as possible toward the right treatments and a cure; to give people more control, empowering them to live life to the full; and last, to achieve consistently high-quality health and social care for everyone affected.

These listening exercises have additionally been backed up by evidence from the 2012 Parkinson's audit [1], feedback from professionals and other surveys of people affected. The 2012 UK-wide Parkinson's audit looked at the provision of care by different professionals involved in managing Parkinson's: elderly care, neurology, occupational therapy, physiotherapy, speech and language therapy as well as comparing the experience of over 4000 patients against national guidelines. The audit found that there were lots of examples of good practice in all the professions. The vast majority of people were reviewed at the right time by a specialist, were on the right medication for their needs and were able to access occupational therapy, physiotherapy and speech and language services at an appropriate time. It is worth noting however, that this was a self-selecting group of participants who are often likely to be the most committed to improving their services.

However, the findings also highlighted some serious shortcomings in the care being offered. For example, there was a widespread lack of information about Parkinson's in clinics in elderly care services and neurology departments. One in 20 people couldn't access a specialist Parkinson's nurse, because a local service was not available - equating to 6350 people across the UK living with Parkinson's without the care and support of a specialist nurse. The audit also showed that, while there was an appetite for collaboration among professionals, many vital services such as occupational therapists, physiotherapists and speech and language therapists were poorly integrated with (primary) clinical services.

The message is clear from people affected by Parkinson's. They want timely, accessible services that anticipate and meet their needs; services to be well integrated with full access to a multidisciplinary team and to be treated as a whole person, not a set of symptoms. They want to be cared for by expert professionals and they want information and opportunities to help make choices, take control and live life to the full. This is at the heart of what the UK Parkinson's Excellence Network [2] will achieve; better services, experiences and outcomes for all our patients (see Figure 1). From my own experience as Director of the Institute of Neuroscience at Newcastle University (UK) I know that networks like this have the potential to make significant positive changes. Over the last 2 years, the Parkinson's team at Newcastle NHS Trust has been changing strategic direction, shifting the focus of the clinic so that it is more patient-focused in a number of ways.

We have moved our clinic to a new building where we have a patient-friendly waiting area; we have improved the signage and addressed logistical issues like parking - all issues that can be a constant frustration to patients. However, most importantly, we have changed our clinical working practice, and brought together a multidisciplinary Parkinson's team in the clinic. We now run what we call a Clinics for Research and Service in Themed Assessment (CRESTA) clinic [3]. It is a one-stop clinic, with a team of consultants, Parkinson's nurses, a physiotherapist and junior doctors - all working alongside one another, so that patients can see the professionals responsible for their care in one place, and so they have fewer appointments. We are also adjacent to and actively involved with our clinical trials unit meaning more patients get the chance to take part in research.

The pertinent point here is that, we are the same people, employing the same skills and experience as before, but we are no longer working in parallel, we are working together. It makes a huge difference to our patient service, and we have been inspired by how dramatically patient satisfaction has improved since we moved to this new model of care through access to a multidisciplinary team and more efficient use of patient time.

The Excellence Network will achieve even more widespread gains for Parkinson's care and support across the UK. I see it as a brilliant opportunity to take the best ideas and practice from professionals all over the country in order to share and disseminate them more widely. In doing so, we will raise the bar so that every person with Parkinson's gets a high-quality standard of care, and as professionals, we can save time and effort by not 'reinventing the wheel'.

This is utilizing the power of networks to enhance clinical practice. In my experience, 
there are a lot of clinicians out there setting up, improving and running great services for their patients. But there are also others who are struggling against bureaucracy, budgets and from a lack of professional support. That is where the Excellence Network comes in. The Excellence Network is about bringing together the voice of people affected by Parkinson's, the resources and infrastructure of Parkinson's UK and the passion and commitment of professionals to work together for change.

\section{Structure of the Excellence Network}

The Excellence Network has six Themed Working Groups, whose function is to focus on improvement in a particular subject area, such as education, evidence-based practice and service development (see Figure 2). Additionally, there are 20 Regional Working Groups, with the remit to empower and support people in their areas to drive change.

Overarching this, there will be an Oversight Group to set the strategy and monitor the success of the Network in driving positive change. Among the evaluation, measures will be the audit results, including outcomes from use of a Patient Response Experience Measure, numbers of people with Parkinson's offered the chance to participate in research and markers such as prescribing patterns across UK regions. The Network will also be able to draw on the valuable advice and experience of an External Advisory Group with international representatives like Olivier Rascol, Professor of Clinical Pharmacology at Toulouse University Hospital, Matthew Stern, Director at the Parkinson's Disease and Movement Disorders Center and Bas Bloem, Medical director, Parkinson Center Nijmegen, as members.

Supporting this structure is a new dedicated infrastructure within Parkinson's UK to oversee the Education, Professional Engagement and Service improvement programs. Integral to the service improvement program will be the ongoing UK wide clinical audit, conducted by Parkinson's UK, which will provide essential data around existing Parkinson's care and allow services to benchmark their performance and impact against this. Service improvement will be tailored to each area of the UK (Northern Ireland, Scotland, Wales and England) in order to drive through the specific changes most needed in that geography.

In order to improve services, we need wellequipped staff; this is where the education

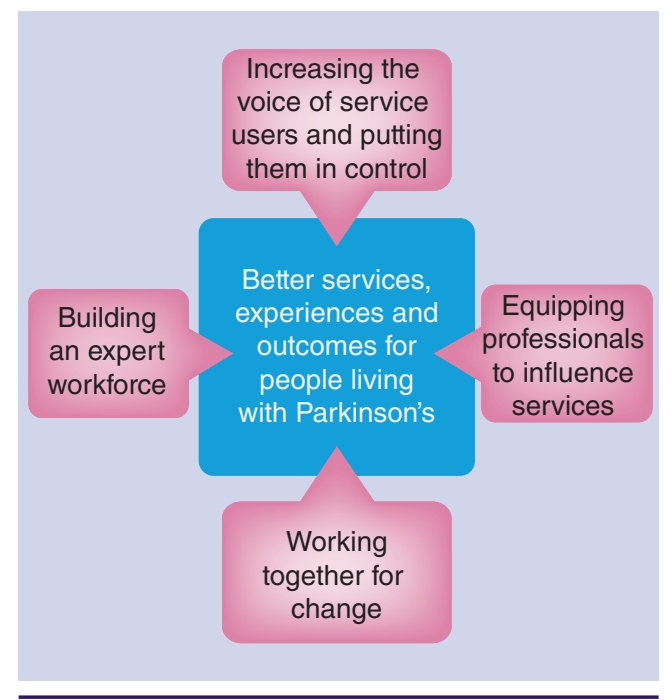

Figure 1. The Excellence Network's aims of better services, experiences and outcomes for people living with Parkinson's will be realized by building an expert workforce, equipping professionals to influence services and increasing the voice of service users and putting them in control.

program is key, providing a range of training options and resources for professionals. The last element of the new structure is professional engagement. Very much a two-way process, this area will focus on how to communicate Excellence Network messaging with professionals, but also how professionals will be best able to share their data and information with the Excellence Network.

\section{Benefits for professionals}

As accessible training and education for Parkinson's was identified as an area of weakness by previous audits [1], the Excellence Network includes an online resource center. This is a one-stop shop for information and tools that can help clinicians drive service improvements, and support professional development. It will also include virtual learning modules to support continued professional development and provide access to a range of best practice guidelines another priority identified by the audit. Moving forward, there will also be new service improvement grants targeted at priority areas and award programs to celebrate best practice.

Additionally, the resource center will carry upto-date details of clinical trials that are recruiting patients in order to make it easier for professionals to find appropriate studies for their patients, 


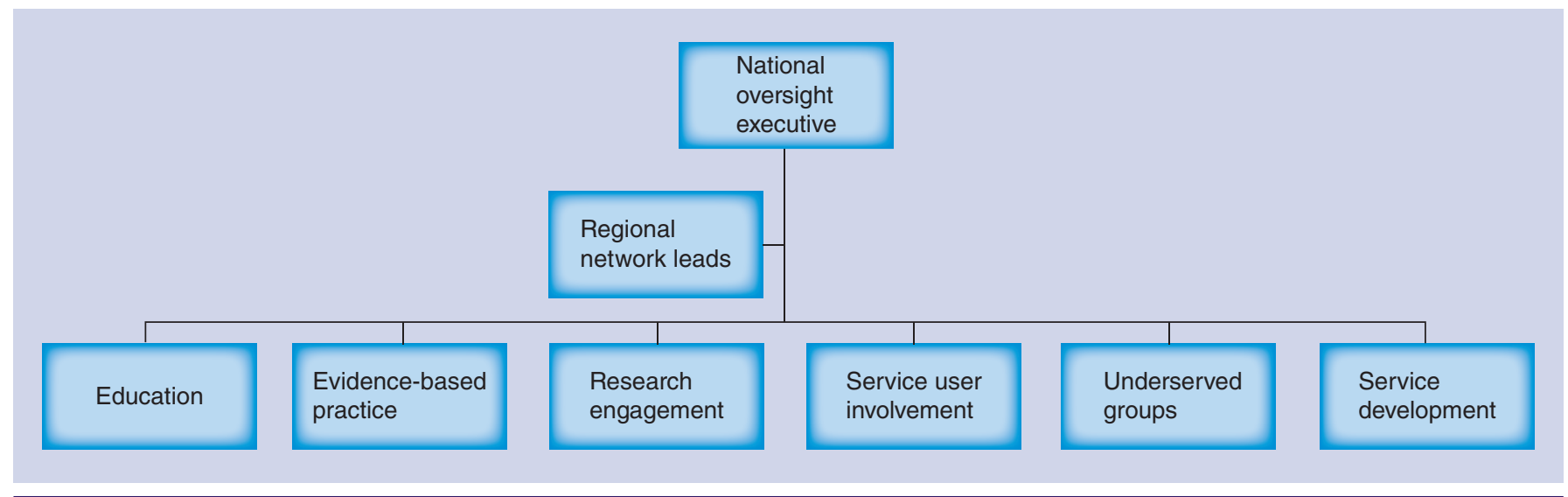

Figure 2. The Excellence Network is structured into six themed working groups, focusing on improvement within their specified area. These themed groups and the regional network leads will be overseen by the National Oversight Executive.

and drive up the number of people involved in clinical trials at any one time. We recognize that best research means best health, so offering more people the chance to participate in research will be a key element of the network.

The Excellence Network will also provide tools to support clinicians in involving people affected by Parkinson's in shaping services as well as providing up-to-date intelligence from across the Parkinson's patient community. There is also a strengthened UK-wide clinical audit process, including a Patient-Reported Experience Measure to evaluate the effectiveness of the work of services from the perspective of the person affected by Parkinson's. Clinicians can access the Excellence Network training resources to fill gaps in knowledge and service provision as well as drawing on individuals and resources in the Excellence Network to influence policy and decisions in their NHS Trust.

Crucially, the Excellence Network is also a way for clinicians to reach out to other professionals locally and across the UK, to share ideas and get support to drive changes in treatment and care.

\section{Future perspective}

Excellence is not an end in itself but a means to an end. That end is first class treatment and care for all people affected by Parkinson's in the
UK. In 2 years' time, I want the Network to have delivered a step change in the provision of first class care for patients. We will only achieve this if clinicians are prepared to get involved and actively encourage colleagues to get involved too. We need clinicians to spread the word about the Excellence Network to everyone involved in Parkinson's care. Additionally, as clinicians we must utilize the Excellence Network and through the UK Parkinson's Audit, benchmark our services, and look for ways to improve.

I would urge anyone involved in the Excellence Network to share their ideas and best practice. Only by working together, we can make a real difference to the quality of patients' lives. With a wealth of clinical commitment, the UK Parkinson's Excellence Network can become a paradigm of excellence in the care of people with this chronic condition.

\section{Financial \& competing interests disclosure}

The authors have no relevant affiliations or financial involvement with any organization or entity with a financial interest in or financial conflict with the subject matter or materials discussed in the manuscript. This includes employment, consultancies, honoraria, stock ownership or options, expert testimony, grants or patents received or pending, or royalties.

No writing assistance was utilized in the production of this manuscript.

\section{References}

1 Parkinson's UK. National Parkinson's Audit (2012). www.parkinsons.org.uk/sites/default/files
2 UK Parkinson's Excellence Network. www.parkinsons.org.uk/excellencenetwork
3 Cresta Clinics, Newcastle University. www.research.ncl.ac.uk/hmst/clinicalteaching 